\title{
Irinotecan-induced ovarian follicular apoptosis is attenuated by deleting the kinase domain of death-associated protein kinase
}

\author{
LI LI $^{1,2}$, TETSUJI TANAKA ${ }^{1}$, KAZUNORI YUKAWA ${ }^{3}$, SHIZUO AKIRA ${ }^{4}$ and NAOHIKO UMESAKI ${ }^{1}$ \\ ${ }^{1}$ Department of Obstetrics and Gynecology, Wakayama Medical University, 811-1 Kimi-idera, Wakayama 641-0012, Japan; \\ ${ }^{2}$ Department of Obstetrics and Gynecology, Qilu Hospital, Shandong University, Jinan 250012, P.R. China; \\ ${ }^{3}$ Department of Physiology, Faculty of Pharmacy, Meijo University, 150 Yagotoyama, Tempaku-ku, \\ Nagoya 468-8503; ${ }^{4}$ Department of Host Defence, Research Institute of Microbial Disease, \\ Osaka University, 3-1 Yamada-oka, Suita, Osaka 565-0871, Japan
}

Received November 24, 2008; Accepted January 20, 2009

DOI: 10.3892/ijo_00000216

\begin{abstract}
Although death-associated protein kinase (DAPK) is a $\mathrm{Ca}^{2+} /$ calmodulin-regulated serine/threonine kinase that plays important roles in various types of apoptotic cell death, there have been no reports of its tissue distributions and functions in female reproductive organs. By comparing C57BL/6 wild-type mice with DAPK-mutant mice lacking the 74-amino acid catalytic kinase domain of DAPK, the cellular distributions and biological functions of DAPK in murine ovaries were investigated. In situ hybridization analyses with sense and antisense riboprobes revealed that DAPK mRNA was selectively and highly expressed in granulosa cells in the ovaries of both types of mice. There were no significant differences in the body weights, ovarian weights and unstimulated ovarian follicular numbers between the wild-type and DAPK-mutant mice. Intraperitoneal injection of CPT-11, an anticancer topoisomerase I inhibitor that causes granulosa cell-specific apoptosis partly through Fas-Fas ligand (FasL) interactions in $\mathrm{MCH}$ mice, induced follicular apoptosis in both the wild-type and DAPK-mutant mice. However, the numbers of apoptotic follicles were significantly reduced in the DAPK-mutant mice. The Fas and FasL expression levels in the CPT-11-injected mice did not differ significantly between the wild-type and DAPK-mutant mice. These results indicate that DAPK positively regulates intracellular signaling pathways for CPT-11-induced granulosa cell apoptosis.
\end{abstract}

\section{Introduction}

Recent combination anticancer chemotherapy regimens have significantly improved the long-term survival rates of advanced

Correspondence to: Dr Tetsuji Tanaka, Department of Obstetrics and Gynecology, Wakayama Medical University, 811-1 Kimi-idera, Wakayama 641-0012, Japan

E-mail: obgywmu@wakayama-med.ac.jp

Key words: apoptosis, granulosa cell, irinotecan, death-associated protein kinase, Fas ligand cancer patients, but can cause irreversible adverse effects (1-3). In young female patients, anticancer chemotherapy sometimes induces severe ovarian toxicity, including infertility and premature ovarian failure. Advances in reproductive technology may provide help toward the preservation of fertility in such patients. Several studies have already evaluated the feasibilities of ovarian protection with concurrent hormone therapy, such as oral contraceptives (OCs) or gonadotropin-releasing hormone agonists (GnRHa), and clinical applications of assisted reproductive technology (ART), including embryo cryopreservation, oocyte cryopreservation, ovarian tissue cryopreservation and in vitro oocyte maturation (3-5). However, ovarian protection with OCs or GnRHa has not been completely established and its effects are currently insufficient. The ART strategies mentioned above are developing technologies and successful pregnancy rates in patients treated with intensive chemotherapy have been low to date $(4,5)$. Although ovarian damage is the main long-term adverse effect following chemotherapy, its molecular mechanisms have not yet been fully elucidated. Therefore, clarification of the mechanisms involved in chemotherapy-induced ovarian toxicity could lead to the development of new strategies for reducing ovarian damage during chemotherapy.

Death-associated protein kinase (DAPK) is a $\mathrm{Ca}^{2+/}$ calmodulin-regulated serine/threonine kinase that plays crucial roles in various types of apoptotic cell death. For example, it is involved in regulating the apoptosis induced by interferon- $\gamma$, tumor necrosis factor- $\alpha$, anti-Fas antibodies and ceramide, and has been implicated as a positive mediator of apoptosis (6-10). However, several reports have shown that DAPK can exert anti-apoptotic actions under certain conditions $(11,12)$. DAPK has a multidomain structure, comprising a kinase domain, eight ankyrin repeats, P-loops, a cytoskeleton-binding region and a death domain (13). Both the kinase domain and death domain are critical for its death-mediating functions $(8,14,15)$. Using DAPK-mutant mice lacking the 74-amino acid catalytic kinase domain of DAPK, we have revealed that DAPK plays a pro-apoptotic role in renal tubular cell apoptosis in chronic obstructive uropathy and renal ischemiareperfusion injuries (16-18). Furthermore, DAPK is involved in modulating spatial memory in mice (19). However, there 
have been no previous studies on the tissue distributions and physiologic functions of DAPK in female reproductive organs. Similarly, there have been no reports regarding DAPK involvement in ovarian follicular apoptosis.

In human and murine ovaries, ovarian follicles develop cyclically and ovulate for possible pregnancy. During normal ovarian follicular development, most developing follicles become apoptotic $(20,21)$, suggesting that ovarian follicles may be very sensitive to apoptotic stimuli. Therefore, anticancer chemotherapy-induced ovarian damage may further enhance the original apoptotic susceptibility of ovarian follicles. The morphological characteristics of apoptosis and positive apoptotic staining have been observed in pregranulosa cells exposed to certain chemotherapeutic agents $(22,23)$. Our previous studies revealed that ovarian dysfunction during chemotherapy with irinotecan $\mathrm{HCl}$ (CPT-11) (24) was caused by specific granulosa cell apoptosis (25). CPT-11, an anticancer prodrug, is converted into a powerful inhibitor of topoisomerase I by carboxyl esterase in the body (26) and is widely used in various cancer chemotherapies. The aims of the present study were to investigate the expression and localization of DAPK in murine ovaries, and to examine whether DAPK is involved in ovarian follicular development and CPT-11-induced ovarian follicular apoptosis.

\section{Materials and methods}

Animals. DAPK-mutant mice expressing a mutant form of DAPK lacking the kinase domain (amino acids 22-95) were generated as described previously (16-19). The mutant mice had a C57BL/6 genetic background, and wild-type littermates were used as controls. The mice were maintained on a 12-h light/12-h dark cycle with free access to food and water in the animal facility of Wakayama Medical University and genotyped by polymerase chain reaction (PCR) analysis of tail DNA (16). All the animal experiments were approved by the Animal Ethics Review Committee of Wakayama Medical University.

Histological evaluation of the number of growing follicles. To examine whether DAPK participates in the development of ovarian follicles, 7-week-old female C57BL/6 wild-type mice $(n=7)$ and DAPK-mutant mice $(n=7)$ were weighed and then sacrificed. Their ovaries were immediately removed, weighed and fixed in $4 \%$ paraformaldehyde solution. The fixed tissues were embedded in Tissue-Tek OCT Compound (Sakura Finetek USA Inc., Torrance, CA), serially sectioned at $5-\mu \mathrm{m}$ thickness and stained with hematoxylin-eosin for microscopic observation. The ovarian sections were sampled and their follicles were counted as described previously (27). Briefly, a stratified sample consisting of every tenth section was used to estimate the numbers of growing follicles. The numbers of growing follicles at different developmental stages were counted in all the selected sections of one ovary and the mean numbers were calculated. The ovarian follicles were classified using the criteria established by Pedersen and Peters (28). Briefly, small follicles were defined as follicles with a single granulosa cell monolayer, medium follicles as those with 2-3 granulosa cell layers and large follicles as those with at least 4 granulosa cell layers. All slides were counted without knowledge of the mouse genotypes.
Reverse transcription $(R T)-P C R$. Total RNA was extracted from ovaries of wild-type and DAPK-mutant mice using an RNeasy Mini Kit (Qiagen Inc., Valencia, CA) according to the manufacturer's instructions. Next, $1 \mu \mathrm{g}$ of total RNA was subjected to RT using a Transcriptor First Strand cDNA Synthesis Kit (Roche Applied Science, Mannheim, Germany) with random primers according to the manufacturer's protocol to create cDNA templates. PCR amplification was performed in a $25-\mu 1$ reaction mixture using a GeneAmp PCR System 9700 Thermocycler (Perkin-Elmer Applied Biosystems, Foster City, CA). The primer sequences used for amplification of the DAPK gene were: forward, 5'-GCAGGAAAACGTGGA CGACT-3'; reverse, 5'-TTGGATCCTTGACCAGCAGT-3' (16). An initial incubation at $95^{\circ} \mathrm{C}$ for 15 min was followed by 35 cycles of denaturation at $94^{\circ} \mathrm{C}$ for $1 \mathrm{~min}$, annealing at $55^{\circ} \mathrm{C}$ for $1 \mathrm{~min}$ and extension at $72^{\circ} \mathrm{C}$ for $1 \mathrm{~min}$, with a final extension step at $72^{\circ} \mathrm{C}$ for $10 \mathrm{~min}(16)$. The $\beta$-actin gene was amplified as a control as described previously (29). The primer sequences used for amplification of the $B$-actin gene were: forward, 5'-GGGCACAGTGTGGGTGAC-3'; reverse, 5'-CTGGCACCACACCTTCTAC-3'.

Western blotting. Total proteins were extracted from ovaries of wild-type and DAPK-mutant mice using T-PER Tissue Protein Extraction Reagent (Pierce, Rockford, IL) containing a protease inhibitor cocktail (Roche Applied Science). The resulting protein concentrations were determined using the Coomassie Plus Reagent (Pierce). Equal amounts of proteins were loaded in the lanes of a $7.5 \%$ polyacrylamide gel, separated by electrophoresis and transferred to a PVDF membrane. After blocking non-specific binding sites with 5\% skim milk, the membrane was incubated with a mouse antiDAPK monoclonal antibody (clone 17; BD Biosciences, San Jose, CA; 1:250 dilution). The antigen-antibody complexes were detected using the ECL-plus Western Blotting System (Amersham Pharmacia Biotech, Buckinghamshire, UK) according to the manufacturer's instructions. An anti- $\beta$-actin antibody (Sigma, St. Louis, MO) was used as a control.

Synthesis of DAPK RNA probes and in situ hybridization. To identify DAPK-expressing cells in murine ovaries, in situ hybridization was performed using digoxigenin (DIG)-labeled riboprobes. A mouse DAPK cDNA fragment (1407 bp) was subcloned into the PCRII-TOPO vector (Invitrogen, Carlsbad, CA), verified by sequencing and used as a template for the synthesis of antisense and sense riboprobes. The obtained riboprobes were labeled with DIG-UTP using a DIG RNA Labeling Kit (SP6/T7; Roche Applied Science) according to the manufacturer's protocol. The labeled riboprobes were fragmented into 100 -base probes by alkaline hydrolysis.

In situ hybridization was performed as previously described (30). Briefly, paraffin sections were prepared from $4 \%$ paraformaldehyde-fixed ovarian tissues from 8-week-old wildtype and DAPK-mutant mice. The sections were hybridized with the DIG-labeled antisense and sense probes for DAPK mRNA for $44 \mathrm{~h}$ at $39^{\circ} \mathrm{C}$ in a hybridization solution, followed by incubation with an anti-DIG-AP antibody (Roche Applied Science; 1:400 dilution) for $2 \mathrm{~h}$ at room temperature. The localizations of the bound probes were visualized by incubation in a nitroblue tetrazolium chloride/5-bromo-4-chloro-3-indolyl phosphate (NBT/BCIP) solution (Roche Applied Science) at 

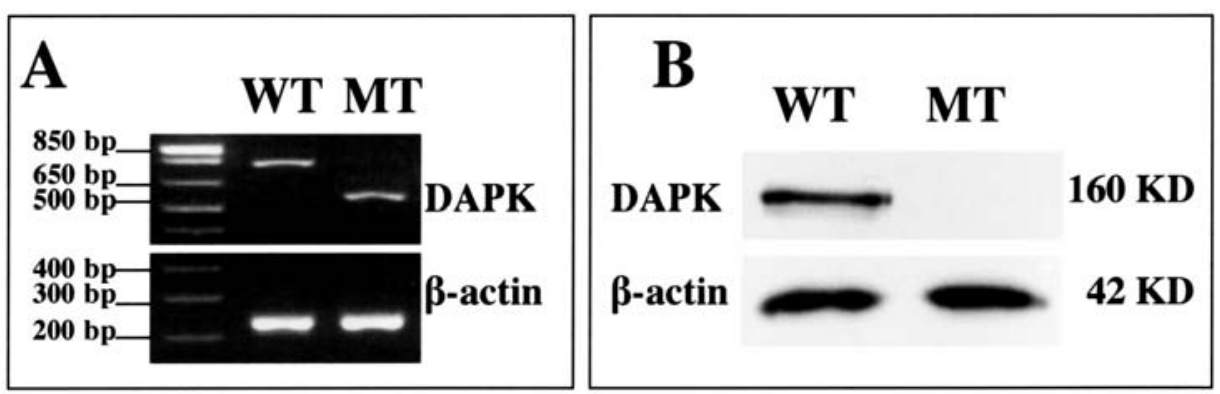

Figure 1. Analyses of DAPK expression in murine ovaries. A, RT-PCR analysis of murine ovaries. A shorter transcript is detected in DAPK-mutant ovaries compared with wild-type ovaries. B, Western blot analysis of murine ovaries using an anti-DAPK monoclonal antibody. A DAPK protein band is detected in the wild-type ovaries. No DAPK protein band is detected in the DAPK-mutant ovaries.

$37^{\circ} \mathrm{C}$ as a substrate. The reaction was stopped by adding $10 \mathrm{mM}$ Tris- $\mathrm{HCl}$ (pH 7.4) containing $1 \mathrm{mM}$ EDTA. The sections were counterstained with Nuclear Fast Red (Vector Laboratories Inc., Burlingame, CA). The sense probe served as a negative control.

Terminal deoxynucleotidyl transferase (TdT)-mediated deoxyUTP nick end labeling (TUNEL) staining of ovarian tissues. A mouse experimental model of CPT-11-induced ovarian injury has recently been established (25). Detection and quantification of CPT-11-induced ovarian follicular apoptosis were performed by in situ TUNEL staining using an ApopTag Peroxidase In Situ Apoptosis Detection Kit (Chemicon International, Temecula, CA) according to the manufacturer's protocol. Eight-week-old female wild-type and DAPK-mutant mice were intraperitoneally injected with CPT-11 (Yakult Co., Tokyo, Japan) at $0.1 \mathrm{mg} / \mathrm{mouse}(\mathrm{n}=5)$ or $0.5 \mathrm{mg} / \mathrm{mouse}$ $(n=6)$. Control mice were intraperitoneally injected with saline $(\mathrm{n}=6)$. The mice were sacrificed at $72 \mathrm{~h}$ after the injection and their ovaries were removed. The ovaries were embedded in Tissue-Tek OCT Compound and cut into 5- $\mu \mathrm{m}$ sections for TUNEL assays and immunohistochemical analyses of Fas and Fas ligand (FasL).

Frozen sections were sequentially fixed with $1 \%$ paraformaldehyde and pre-cooled ethanol:acetic acid. After washing with phosphate-buffered saline (PBS), endogenous peroxidase activity was inhibited by treatment with $3 \% \mathrm{H}_{2} \mathrm{O}_{2}$ solution for $5 \mathrm{~min}$ at room temperature. After soaking with the equilibration buffer of the ApopTag kit, the sections were incubated with the TdT reaction mixture in a humidified chamber at $37^{\circ} \mathrm{C}$ for $1 \mathrm{~h}$. The reaction was terminated by incubation with the stop buffer of the ApopTag kit, and the sections were washed with PBS. The sections were then incubated with a horseradish peroxidase-conjugated anti-DIG antibody at room temperature for $30 \mathrm{~min}$. A color reaction was produced with $0.05 \%$ 3,3-diaminobenzidine-tetrahydrochloride (DAB; Dako, Carpinteria, CA) in $0.01 \% \mathrm{H}_{2} \mathrm{O}_{2}$ solution. The sections were counterstained with methyl green. Classification and nomination of the ovarian follicles were carried out as described above. TUNEL-positive follicles were determined as follicles containing at least 10 TUNELpositive cells within an ovarian follicular section (25).

Immunohistochemical studies of ovaries from CPT-11-injected mice. Frozen ovarian sections from the CPT-11-injected mice were prepared as described above, dried at room temperature, fixed with acetone at $4^{\circ} \mathrm{C}$ for 10 min and washed with PBS. After quenching the endogenous peroxidase activity with $3 \%$ $\mathrm{H}_{2} \mathrm{O}_{2}$ for $10 \mathrm{~min}$ and washing with PBS, the sections were treated with $5 \%$ normal goat serum at room temperature for $1 \mathrm{~h}$ to block non-specific binding. The sections were then incubated with rabbit polyclonal antibodies against Fas or FasL (Santa Cruz Biotechnology, Santa Cruz, CA) overnight at $4^{\circ} \mathrm{C}$. After washing with PBS, the sections were incubated with a peroxidase-conjugated anti-rabbit secondary antibody (Dako) at room temperature for $30 \mathrm{~min}$, washed and reacted with $0.5 \mathrm{mg} / \mathrm{ml} \mathrm{DAB}$ in $10 \mathrm{mM}$ Tris- $\mathrm{HCl}$ (pH 7.6) buffer containing $0.01 \% \mathrm{H}_{2} \mathrm{O}_{2}$ for antigen detection. The sections were counterstained with hematoxylin. The expression levels of Fas and FasL were compared among the different groups.

Statistical analyses. All graphical data were expressed as the mean \pm SE. Data comparisons were analyzed by t-tests and/ or one-way ANOVA. Values of $\mathrm{p}<0.05$ were considered to indicate statistical significance.

\section{Results}

Expressions of DAPK $m R N A$ and protein in ovaries. First, DAPK mRNA and protein expressions were examined in ovaries from wild-type and DAPK-mutant mice. As shown in Fig. 1, RT-PCR and Western blot analyses detected DAPK mRNA and protein, respectively, in the wild-type ovaries. RT-PCR analysis of the DAPK-mutant ovaries identified a shorter transcript compared with that detected in the wild-type ovaries (Fig. 1A). The size of the shorter mRNA coincided with the estimated length of the mutant DAPK mRNA lacking the kinase domain. Notably, no DAPK-specific protein band was detected in the DAPK-mutant ovaries by Western blot analysis. Specifically, a single $160-\mathrm{kDa}$ protein band was detected in the wild-type ovaries but there was no shorter DAPK protein band in the DAPK-mutant ovaries (Fig. 1B).

Localizations of DAPK $m R N A$ in ovarian tissues. As described above, DAPK mRNA was expressed in both wild-type and DAPK-mutant ovaries, whereas DAPK protein was detected in wild-type ovaries but not in DAPK-mutant ovaries. Since there were no anti-DAPK antibodies available for immunohistochemical studies of murine tissues, we performed in situ hybridization for DAPK mRNA expression to identify the DAPK-expressing cells in the ovaries. Although DAPK mRNA was detected extensively but very weakly in all types of ovarian 
WT

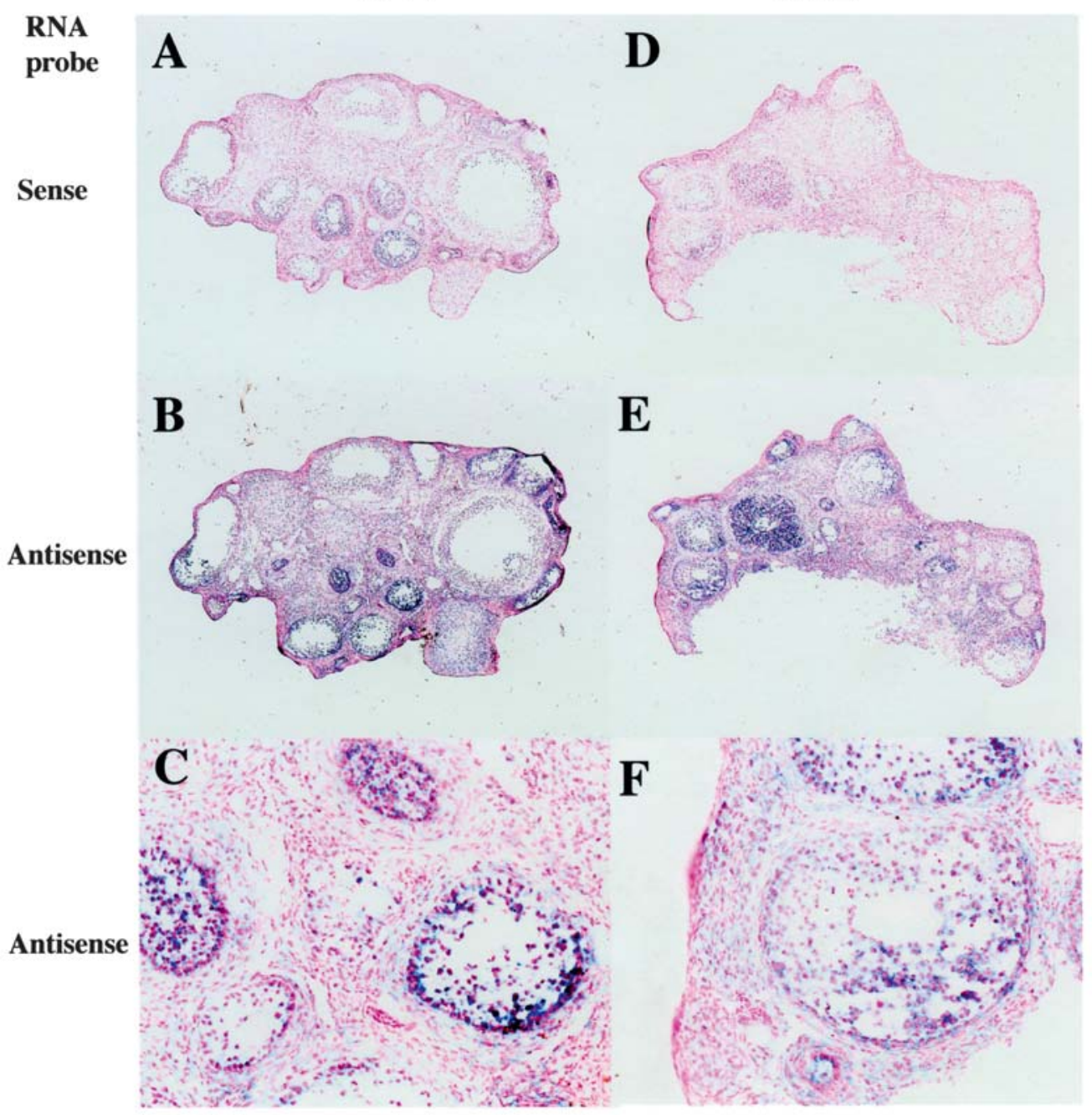

Figure 2. In situ hybridization analysis of DAPK mRNA in murine ovarian tissues. A and D, In situ hybridization with sense riboprobes for DAPK as negative controls. B, C, E and F, In situ hybridization with antisense riboprobes for DAPK showing DAPK mRNA localization in ovarian granulosa cells. C and F, are magnified views of B and E, respectively. WT, wild-type mice; MT, DAPK-mutant mice.
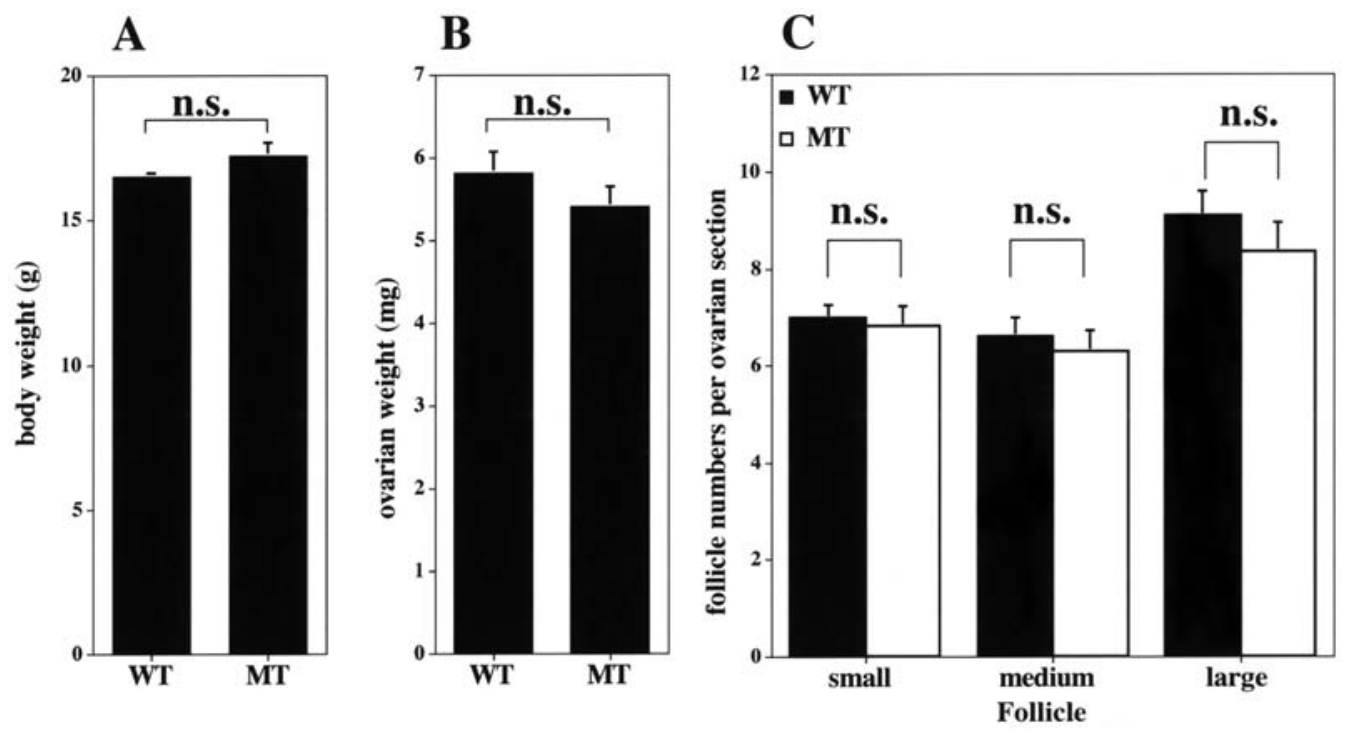

Figure 3. Comparisons of wild-type and DAPK-mutant mice. A, Body weights of 7-week-old female mice. B, Ovarian weights of 7-week-old female mice. C, Ovarian follicular numbers in 7-week-old female mice. The mean numbers of ovarian follicles per ovarian section were compared between the two types of mice. There are no significant differences in the body weights, ovarian weights and ovarian follicular numbers between the wild-type and DAPK-mutant mice. WT, wildtype mice; MT, DAPK-mutant mice; ns, not significant. 


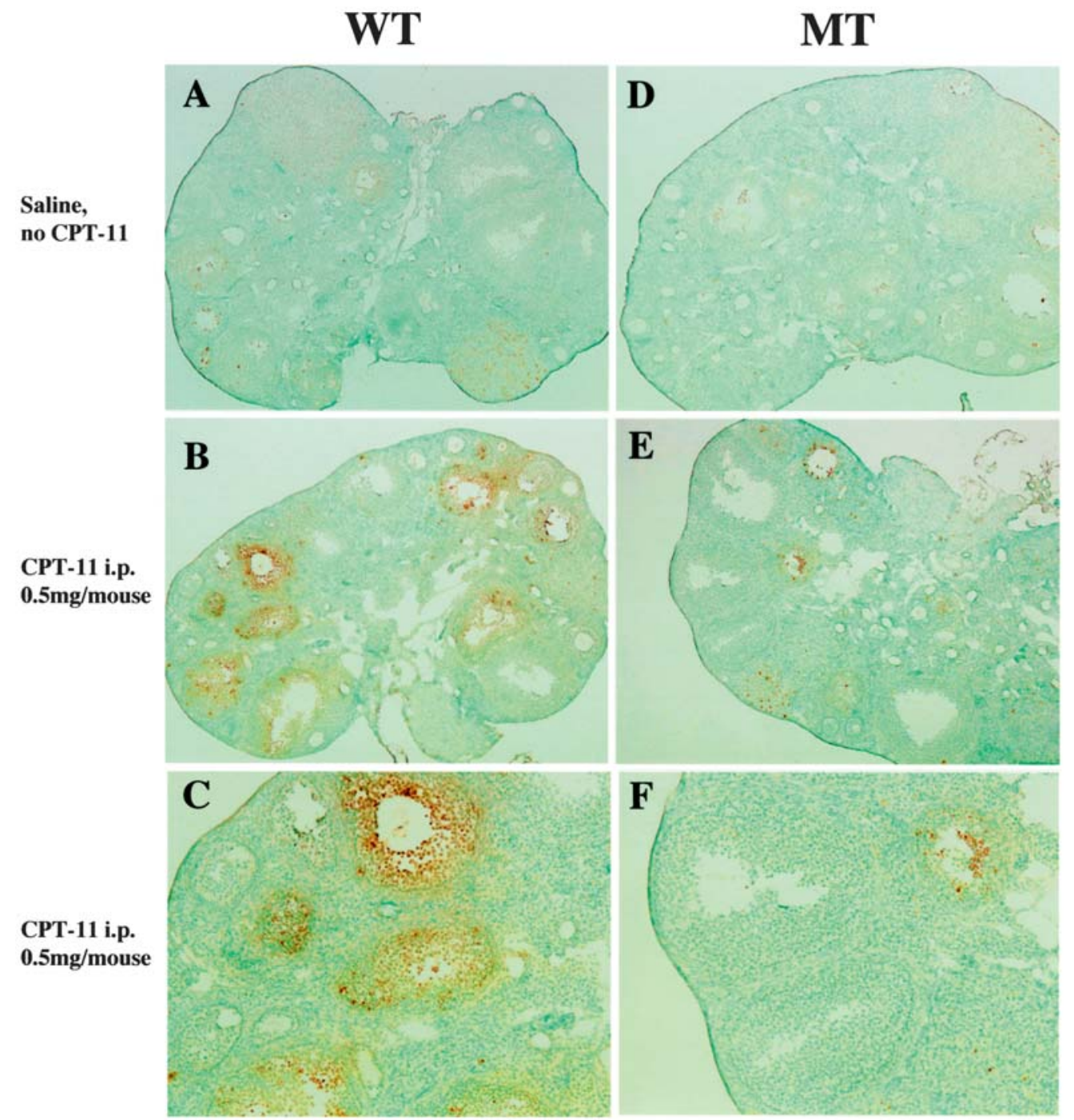

Figure 4. TUNEL staining analysis of ovaries from CPT-11-injected mice. A and D, Ovaries from the saline-injected mice as negative controls. B, C, E and F, Ovaries from the CPT-11-injected mice. C and F, are magnified views of B and E, respectively. WT, wild-type mice; MT, DAPK-mutant mice.

cells in both wild-type and DAPK-mutant mice, high levels of DAPK mRNA transcripts were predominantly localized in the granulosa cells in all stages of follicles and atretic follicles (Fig. 2). In all stages of healthy follicles in both wild-type and DAPK-mutant mice, the expression levels of DAPK mRNA were much higher in large follicles than in small and medium follicles. No differences in the DAPK mRNA expression patterns were observed between the wild-type and DAPKmutant mice.

Body weights, ovarian weights and ovarian follicle counts in wild-type and DAPK-mutant mice. Since DAPK is highly expressed in large follicular granulosa cells (Fig. 2), which comprise the major estrogen-producing cells, it was possible that there were significant differences in the ovarian follicular numbers, ovarian weights and body developments between the wild-type and DAPK-mutant mice. Comparisons between the wild-type and DAPK-mutant mice revealed no significant differences in their body weights (Fig. 3A) or ovarian weights (Fig. 3B).

To examine whether the deletion of the DAPK kinase domain affected folliculogenesis, the numbers of growing follicles were counted according to the criteria described in the Materials and methods. As shown in Fig. 3C, there were no significant differences in the numbers of large follicles, medium follicles and small follicles between the wild-type and DAPK-mutant mice.

These results suggest that the wild-type and DAPK-mutant mice were well matched in their body weights, ovarian weights and follicle numbers for use in for the following CPT-11 injection experiments.

CPT-11-induced follicular apoptosis in ovaries from wildtype and DAPK-mutant mice. As described above, the in situ hybridization analyses revealed that DAPK is highly and specifically expressed in large follicular granulosa cells (Fig. 2). These findings indicate that DAPK may regulate granulosa cell-specific apoptosis. CPT-11 induces follicular apoptosis in murine and human ovaries $(24,25)$. Therefore, to examine whether DAPK plays roles in CPT-11-induced follicular apoptosis, the effects of CPT-11 injection on ovarian follicles were evaluated in the wild-type and DAPK-mutant ovaries. As shown in Fig. 4, an intraperitoneal injection of CPT-11 apparently increased the numbers of TUNEL-positive granulosa cells in both the wild-type and DAPK-mutant mice. The TUNEL-reactivity of the granulosa cells in the CPT-11- 

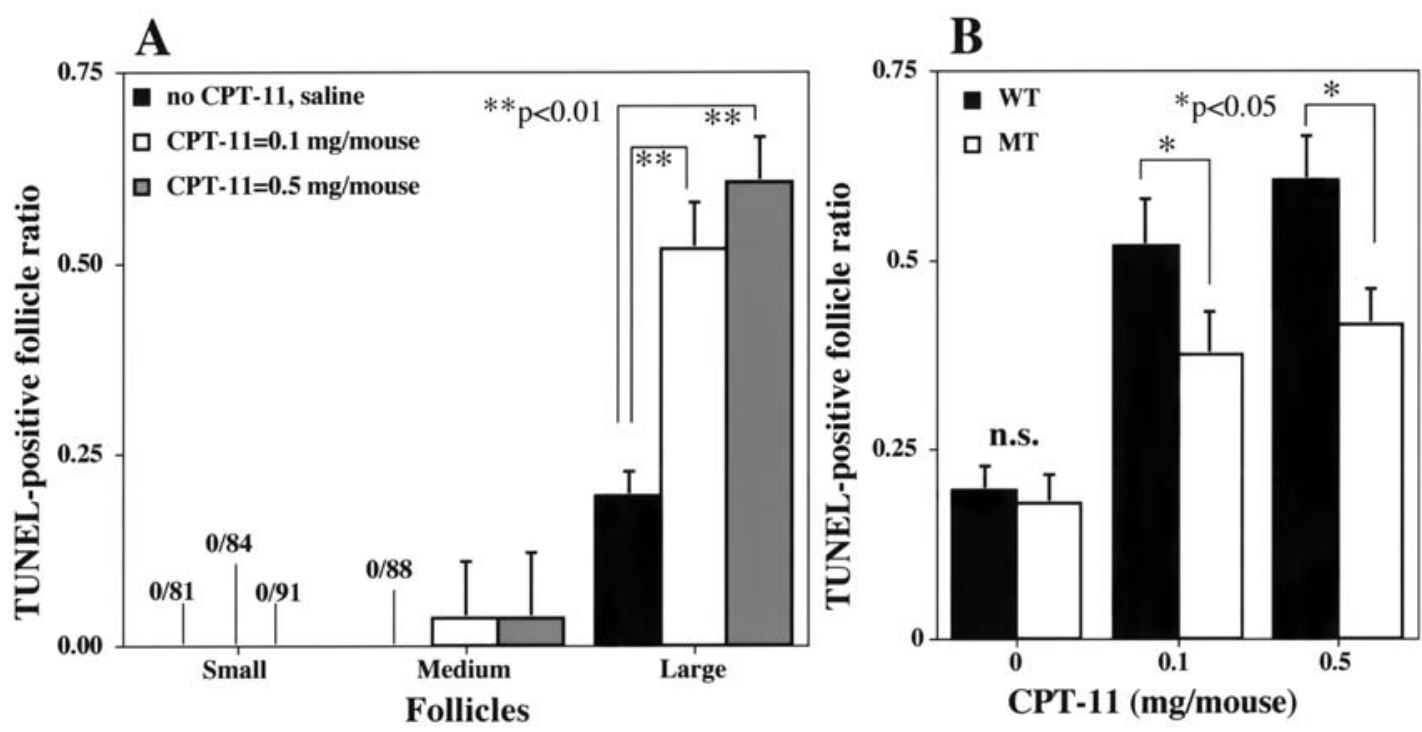

Figure 5. TUNEL-positive follicle ratios in ovaries from CPT-11-injected mice. A, The TUNEL-positive follicle ratios of the small ovarian follicles, medium ovarian follicles and large ovarian follicles in the CPT-11-injected wild-type mice were counted. CPT-11 injection increases dose-dependently the TUNEL-positive follicle ratios of the large ovarian follicles. No TUNEL-positive follicles were detected among 81 small follicles from ten saline-injected murine ovaries, among 84 small follicles from ten $0.1 \mathrm{mg}$ - CPT-11-injected murine ovaries and among 91 small follicles from twelve 0.5 mg - CPT-11-injected murine ovaries. No TUNEL-positive follicles were also detected among 88 medium follicles from ten saline-injected murine ovaries. B, CPT-11-induced TUNEL-positive follicle ratio is significantly and partially reduced in the DAPK-mutant ovaries compared with the wild-type ovaries. WT, wild-type mice; MT, DAPK-mutant mice; ns, not significant. ${ }^{*} \mathrm{p}<0.05,{ }^{* *} \mathrm{p}<0.01$.

injected wild-type mice was much stronger than that in the CPT-11-injected DAPK-mutant mice (Fig. 4B and E). When the follicles containing at least 10 TUNEL-positive granulosa cells in the ovarian sections were defined as TUNEL-positive follicles, TUNEL-positive follicles were mainly detected in large follicles in CPT-11-injected wild-type and DAPK-mutant mice (Figs. 4 and 5), but rarely detected in small and medium follicles, suggesting that large follicles were more sensitive to CPT-11-induced follicular apoptosis. These findings are consistent with those in a previous report using a different mouse strain (25). Notably, however, the TUNEL-positive large follicle ratios were significantly higher in the wild-type mice than in the DAPK-mutant mice (Fig. 5B).

Expression of Fas and FasL in ovaries from the CPT-11injected mice. Our previous studies indicated that follicular apoptosis through Fas-FasL interactions in granulosa cells was one of the molecular mechanisms involved in CPT-11induced ovarian failure $(24,25)$. Therefore, we performed immunohistochemical analyses to examine whether the different apoptotic susceptibilities of ovarian follicles stimulated by CPT-11 between the wild-type and DAPKmutant mice was caused by differential inductions of Fas and/or FasL expressions in granulosa cells. In the salineinjected control mice without CPT-11 administration, Fas expression was similarly detected in almost all types of ovarian cells in the wild-type and DAPK-mutant mice, including granulosa cells (Fig. 6A and C). In the CPT-11-injected mice, the tissue distributions and immunoreactivities of Fas in the ovaries showed no apparent differences between the wildtype and DAPK-mutant mice (Fig. 6B and D).

In the saline-injected control mice, small numbers of large and medium follicles showed immunoreactivities for FasL in both the wild-type and DAPK-mutant mice, while the extrafollicular ovarian cells did not show any FasL expression
(Fig. 6E and G). In the CPT-11-injected mice, higher levels of FasL immunoreactivity were detected in the granulosa cells in most large follicles and several medium follicles in both the wild-type and DAPK-mutant mice (Fig. $6 \mathrm{~F}$ and $\mathrm{H}$ ), while no FasL expression was found in small follicles. When the follicles containing at least 10 strongly FasL-positive cells per ovarian section were determined as FasL-positive follicles (25), the FasL-positive follicle ratios of medium and large follicles in the CPT-11-injected mice $(0.1 \mathrm{mg}$ and $0.5 \mathrm{mg} /$ mouse) were significantly higher than those in the salineinjected control mice (Fig. 7A). However, no FasL-positive follicle was detected in the small follicles. CPT-11 dosedependently increased FasL-positive follicle ratios of medium follicles (Fig. 7B) and large follicles (Fig. 7C) in both types of mice. In contrast, in both the saline-injected control and CPT-11-injected mice, there were no significant differences in the FasL-positive follicle ratios between the wild-type and DAPK-mutant mice (Fig. 7B and C).

\section{Discussion}

Since DAPK was initially identified as a positive mediator of interferon- $\gamma$-induced programmed cell death in the HeLa human cervical cancer cell line (6), the apoptosis-related functions of DAPK products have been extensively investigated using various types of in vitro-cultured cells (7-10). However, the physiological functions of DAPK in vivo were not fully understood until DAPK-mutant mice were generated. Although a few studies have shown roles for DAPK in the kidney and brain (16-19), the functions of DAPK in the ovary have not previously been reported. In the present study, we have clarified the tissue distributions and biocharacteristics of DAPK in the murine ovary for the first time. By using a DAPK-mutant mouse model, the biological functions of DAPK in ovarian follicular growth and its involvement in CPT-11- 


\section{Saline i.p., no CPT-11 CPT-11 i.p., 0.5mg/mouse}

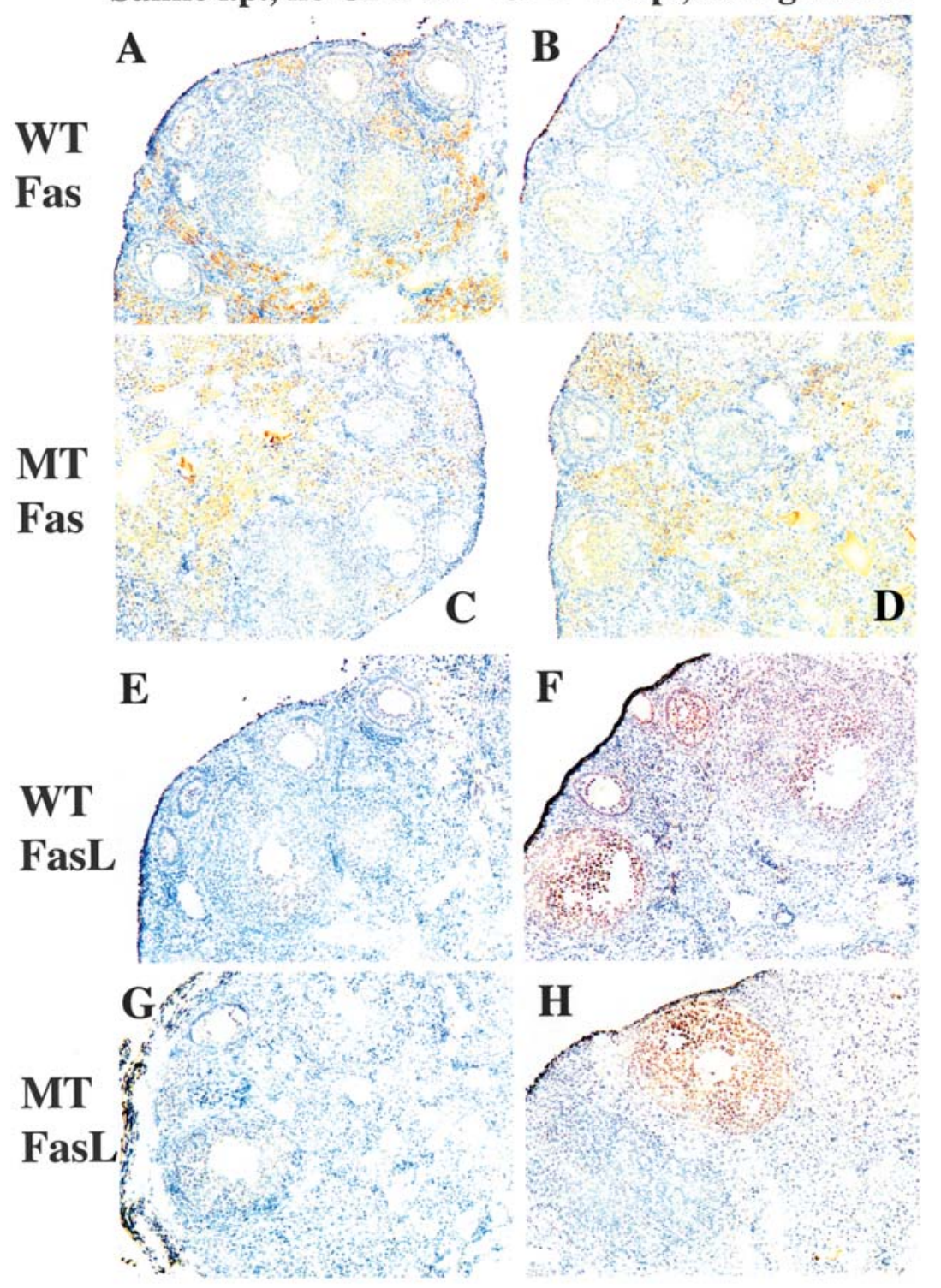

Figure 6. Immunohistochemical analyses of Fas and FasL expression in ovaries from CPT-11-treated wild-type and DAPK-mutant mice. A-D, Immunohistochemical analyses of Fas expression in ovarian tissues. There are no apparent differences in the Fas expression patterns between the wild-type and DAPK-mutant mice. E-H, Immunohistochemical studies of FasL expression in ovarian tissues. There are no apparent differences in the FasL expression patterns between the wild-type and DAPK-mutant mice. WT, wild-type mice; MT, DAPK-mutant mice.

induced ovarian follicular apoptosis were investigated. We found that deletion of the DAPK kinase domain significantly attenuated ovarian follicular apoptosis in CPT-11-injected mice in vivo, suggesting that DAPK may act as a positive modulator of granulosa cell apoptosis in certain types of anticancer drug-induced ovarian failure.

DAPK is widely expressed in many kinds of cells and tissues, implying that it plays roles in various types of apoptotic cell death (6-10). DAPK protein expression was previously detected in normal human endometrium, normal murine ovary and normal murine uterus by Western blot analyses (31). However, there have been no reports about the tissue localizations and functions of DAPK in female genital organs, particularly in the context of ovarian follicular development and apoptosis. To understand this issue, we first examined the tissue localization of DAPK in murine ovaries. RT-PCR analyses of the DAPK-mutant ovaries detected the expression of a DAPK-mutant mRNA that was shorter than the wildtype transcript, as previously shown in DAPK-mutant mouse kidneys $(16,17)$. However, Western blot analysis did not detect a corresponding DAPK protein band in the DAPK-mutant ovaries. These results indicate that deletion of the DAPK kinase domain may have caused certain conformational changes in the mutant DAPK protein, rendering the mutant protein unrecognizable by the anti-DAPK monoclonal antibody used in the Western blotting analysis. Alternatively, deletion of the DAPK kinase domain may have blocked DAPK mRNA translation, thereby inhibiting DAPK protein expression in the mouse ovaries. Suppressed translation of DAPK mRNA is often observed in cancer cells because DAPK protein expression can be regulated by the methylation status of the promoter region of the DAPK gene $(31,32)$. Therefore, the lack of detectable mutant DAPK protein in the mouse ovary in the present study may have been caused by a 

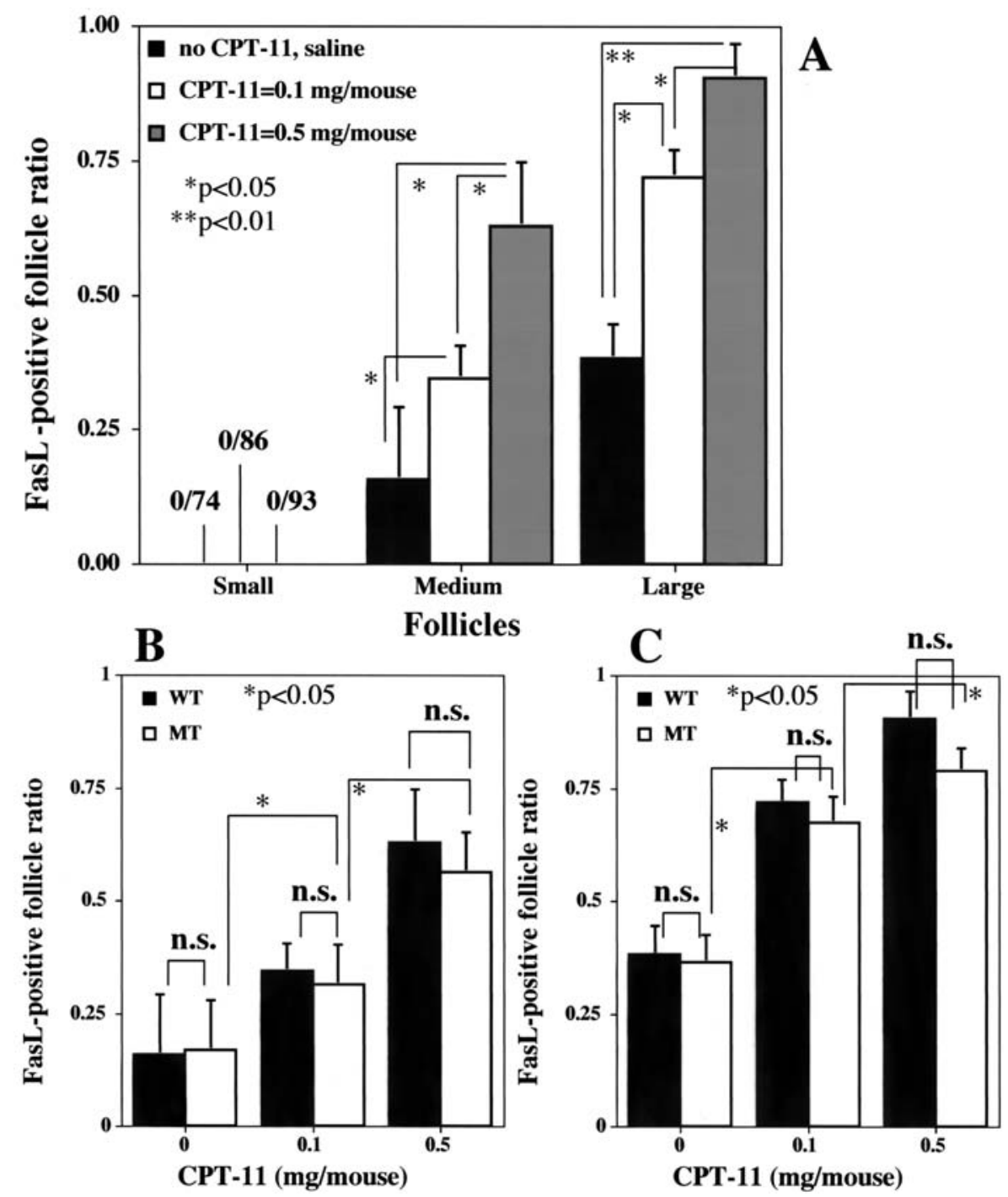

Figure 7. FasL-positive follicle ratios in the murine ovaries. A, The FasL-positive follicle ratios of the small ovarian follicles, medium ovarian follicles and large ovarian follicles in the CPT-11-injected wild-type mice were counted. CPT-11 dose-dependently induces FasL expression in medium follicles and large follicles in the murine ovaries. No FasL-positive follicles were detected among 74 small follicles from ten saline-injected murine ovaries, among 86 small follicles from ten $0.1 \mathrm{mg}$ - CPT-11-injected murine ovaries and among 93 small follicles from twelve $0.5 \mathrm{mg}$ - CPT-11-injected murine ovaries. B, The FasL-positive medium follicle ratios in the CPT-11-injected mice were compared. There are no significant differences in the FasL-positive medium follicle ratios between the wild-type and DAPK-mutant mice. C, The FasL-positive large follicle ratios in the CPT-11-injected mice were compared. There are no significant differences in the FasLpositive large follicle ratios between the wild-type and DAPK-mutant mice. WT, wild-type mice; MT, DAPK-mutant mice; ns, not significant. ${ }^{*} \mathrm{p}<0.05,{ }^{* *} \mathrm{p}<0.01$.

mechanism involving DAPK gene promoter methylation. Another possibility is that the defective DAPK mRNA may be translated into a very unstable DAPK protein by an unknown mechanism.

Some anticancer drugs are well known to induce ovarian damage in addition to cancer cell death (1-5). Anticancer drugs may increase the apoptotic susceptibilities of normal ovarian follicles and cause granulosa cell apoptosis in ovarian follicles. Recently, we reported that intraperitoneal injection of CPT-11 induces FasL expression in the granulosa cells of large follicles and that apoptosis is induced in these granulosa cells by interactions of FasL and Fas constitutively expressed in granulosa cells (25). Nevertheless, the possible induction of other intracellular signaling pathways for granulosa cell apoptosis by anticancer drugs has hardly been investigated. The numbers of ovarian follicles at each developmental stage and the ovarian weights of the DAPK-mutant mice were similar to those in the wild-type mice. These findings indicate that DAPK has no significant effects on ovarian follicular development under normal conditions and that these types of mice were well matched for investigations of DAPK functions in anticancer drug-induced ovarian damage. Our in situ hybridization assays revealed that DAPK mRNA was highly expressed in ovarian granulosa cells, but widely and very weakly distributed in various ovarian cells, suggesting that DAPK may play granulosa cell-specific roles in the ovary. Further experiments demonstrated that CPT-11 could induce granulosa cell apoptosis, mainly in large follicles, in both the wild-type and DAPK-mutant mice, although the follicular apoptosis was significantly fewer and much weaker in the DAPK-mutant mice than in the wild-type mice. These findings indicate that deletion of the DAPK kinase domain significantly attenuates CPT-11-induced granulosa cell apoptosis.

Fas is a transmembrane glycoprotein that belongs to the tumor necrosis factor receptor family and mediates apoptosis in many cell types $(33,34)$. FasL, a well-known cell death 
factor and a type II integral membrane protein homologous to tumor necrosis factor (35), specifically binds to Fas and induces cell death through apoptosis (36). Our previous study confirmed that CPT-11-induced FasL expression in granulosa cells is one of the main mechanisms for CPT-11-induced ovarian follicular apoptosis in mice (25). Therefore, to examine whether DAPK is involved in the increase in FasL expression during CPT-11-induced follicular apoptosis, the expression levels of Fas and FasL in the follicles after CPT-11 injection were compared between the wild-type and DAPK-mutant mice. The Fas expression levels were similar in the wild-type and DAPK-mutant mice, regardless of whether or not they received CPT-11 treatment. Moreover, in the CPT-11-injected mice, there were no significant differences in the FasLpositive follicular ratios between the wild-type and DAPKmutant mice, but the follicular apoptosis was much stronger in the wild-type mice than in the DAPK-mutant mice. These results suggest that DAPK activity may be involved in an apoptotic signaling pathway distinct from the regulation of FasL expression in CPT-11-induced follicular apoptosis.

The present study has revealed a crucial role for DAPK in CPT-11-induced ovarian follicular apoptosis. However, the follicular apoptosis was not completely inhibited in the DAPKmutant mice. These findings suggest that follicular apoptosis is regulated by several complex systems involving large numbers of molecules and that DAPK-mediated signaling is only part of the regulatory system. The existence of such DAPK-independent regulatory signals in follicular apoptosis may represent biological redundancy for reproduction to avoid complete ovarian damage.

In summary, the present study has revealed that deletion of the DAPK kinase domain does not affect normal follicular development, despite the fact that DAPK mRNA is highly expressed in ovarian granulosa cells, but significantly attenuates the ovarian follicular apoptosis induced by CPT-11 in vivo. The present results indicate that DAPK positively regulates the ovarian follicular apoptosis activated by anticancer drugs. Therefore, DAPK activity in ovarian follicles may represent one of the molecular mechanisms involved in anticancer drug-induced ovarian failure. The present findings also suggest that DAPK may play a physiological role in the induction of follicular apoptosis to remove ovarian follicles damaged by chemicals and maintain normal reproduction. On the other hand, molecular targeting therapies that specifically inhibit DAPK activity may reduce the ovarian follicular loss induced by some anticancer drugs and preserve the reproductive capability of young female cancer patients. Finally, DAPKmutant mice may be useful for investigations of the various biological functions of DAPK and ovarian follicular apoptosis.

\section{Acknowledgements}

This study was supported in part by a Grant-in-Aid for scientific research from the Ministry of Education, Science, Sports and Culture of Japan.

\section{References}

1. Meirow D and Nugent D: The effects of radiotherapy and chemotherapy on female reproduction. Hum Reprod Update 7: 535-543, 2001.
2. Fossa SD and Magelssen H: Fertility and reproduction after chemotherapy of adult cancer patients: malignant lymphoma and testicular cancer. Ann Oncol 15 (Suppl): 259-265, 2004.

3. Lutchman SK, Davies M and Chatterjee R: Fertility in female cancer survivors: pathophysiology, preservation and the role of ovarian reserve testing. Hum Reprod Update 11: 69-89, 2005.

4. Davis VJ: Female gamete preservation. Cancer 107: 1690-1694, 2006.

5. Stern CJ, Toledo MG, Gook DA and Seymour JF: Fertility preservation in female oncology patients. Aust N Z J Obstet Gynaecol 46: 15-23, 2006.

6. Deiss LP, Feinstein E, Berissi H, Cohen O and Kimchi A: Identification of a novel serine/threonine kinase and a novel $15-\mathrm{kD}$ protein as potential mediators of the gamma interferoninduced cell death. Genes Dev 9: 15-30, 1995.

7. Inbal B, Cohen O, Polak-Charcon S, Kopolovic J, Vadai E, Eisenbach L and Kimchi A: DAP kinase links the control of apoptosis to metastasis. Nature 390: 180-184, 1997.

8. Cohen O, Feinstein E and Kimchi A: DAP-kinase is a $\mathrm{Ca} 2+/$ calmodulin-dependent, cytoskeletal-associated protein kinase, with cell death-inducing functions that depend on its catalytic activity. EMBO J 16: 998-1008, 1997.

9. Cohen O, Inbal B, Kissil JL, Raveh T, Berissi H, SpivakKroizaman T, Feinstein E and Kimchi A: DAP-kinase participates in TNF-alpha- and Fas-induced apoptosis and its function requires the death domain. J Cell Biol 146: 141-148, 1999.

10. Pelled D, Raveh T, Riebeling C, Fridkin M, Berissi H, Futerman AH and Kimchi A: Death-associated protein (DAP) kinase plays a central role in ceramide-induced apoptosis in cultured hippocampal neurons. J Biol Chem 277: 1957-1961, 2002.

11. Jin Y, Blue EK, Dixon S, Hou L, Wysolmerski RB and Gallagher PJ: Identification of a new form of death-associated protein kinase that promotes cell survival. J Biol Chem 276: 39667-39678, 2001.

12. Jin Y, Blue EK, Dixon S, Shao Z and Gallagher PJ: A deathassociated protein kinase (DAPK)-interacting protein, DIP-1, is an E3 ubiquitin ligase that promotes tumor necrosis factor-induced apoptosis and regulates the cellular levels of DAPK. J Biol Chem 277: 46980-46986, 2002.

13. Tereshko V, Teplova M, Brunzelle J, Watterson DM and Egli M: Crystal structures of the catalytic domain of human protein kinase associated with apoptosis and tumor suppression. Nat Struct Biol 8: 899-907, 2001

14. Kimchi A: DAP kinase and DAP-3: novel positive mediators of apoptosis. Ann Rheum Dis 58 (Suppl 1): 114-119, 1999.

15. Bialik S and Kimchi A: The death-associated protein kinases: structure, function, and beyond. Annu Rev Biochem 75: 189-210, 2006.

16. Yukawa K, Hoshino K, Kishino M, Mune M, Shirasawa N, Kimura A, Tsubota Y, Owada-Makabe K, Tanaka T, Ichinose M, Maeda M, Takeda K and Akira S: Deletion of the kinase domain in death-associated protein kinase attenuates renal tubular cell apoptosis in chronic obstructive uropathy. Int J Mol Med 13: 515-520, 2004.

17. Kishino M, Yukawa K, Hoshino K, Kimura A, Shirasawa N, Otani H, Tanaka T, Owada-Makabe K, Tsubota Y, Maeda M, Ichinose M, Takeda K, Akira S and Mune M: Deletion of the kinase domain in death-associated protein kinase attenuates tubular cell apoptosis in renal ischemia-reperfusion injury. J Am Soc Nephrol 15: 1826-1834, 2004.

18. Yukawa K, Hoshino K, Kishino M, Tsubota Y, Owada-Makabe K, Maeda M, Bai T, Tanaka T and Akira S: Deletion of the kinase domain from death-associated protein kinase attenuates p53 expression in chronic obstructive uropathy. Int J Mol Med 16: 389-393, 2005.

19. Yukawa K, Tanaka T, Bai T, Li L, Tsubota Y, Owada-Makabe K, Maeda M, Hoshino K, Akira S and Iso H: Deletion of the kinase domain from death-associated protein kinase enhances spatial memory in mice. Int J Mol Med 17: 869-873, 2006.

20. Fulton N, Martins da Silva SJ, Bayne RA and Anderson RA: Germ cell proliferation and apoptosis in the developing human ovary. J Clin Endocrinol Metab 90: 4664-4670, 2005.

21. Hussein MR: Apotosis in the ovary: molecular mechanisms. Hum Reprod Update 11: 162-177, 2005.

22. Familiari G, Caggiati A, Nottola SA, Ermini M, Di Benedetto MR and Motta PM: Ultrastructure of human ovarian primordial follicles after combination chemotherapy for Hodgkin's disease. Hum Reprod 8: 2080-2087, 1993. 
23. Perez GI, Knudson CM, Leykin L, Korsmeyer SJ and Tilly JL: Apoptosis-associated signaling pathways are required for chemotherapy-mediated female germ cell destruction. Nat Med 3: 1228-1232, 1997.

24. Tanaka $\mathrm{T}$, Utsunomiya $\mathrm{T}$, Utsunomiya $\mathrm{H}$ and Umesaki $\mathrm{N}$ : Irinotecan $\mathrm{HCL}$, an anticancer topoisomerase I inhibitor, frequently induces ovarian failure in premenopausal and perimenopausal women. Oncol Rep 19: 1123-1133, 2008.

25. Utsunomiya $T$, Tanaka $T$, Utsunomiya $H$ and Umesaki $\mathrm{N}$ : A novel molecular mechanism for anticancer drug-induced ovarian failure: Irinotecan $\mathrm{HCl}$, an anticancer topoisomerase I inhibitor, induces specific FasL expression in granulosa cells of large ovarian follicles to enhance follicular apoptosis. Int J Oncol 32: 991-1000, 2008

26. Kawato $\mathrm{Y}$, Aonuma M, Hirota $\mathrm{Y}$, Kuga $\mathrm{H}$ and Sato K: Intracellular roles of $\mathrm{SN}-38$, a metabolite of the camptothecin derivative CPT-11, in the antitumor effect of CPT-11. Cancer Res 51: 4187-4191, 1991.

27. Borgeest C, Miller KP, Gupta R, Greenfeld C, Hruska KS, Hoyer P and Flaws JA: Methoxychlor-induced atresia in the mouse involves Bcl-2 family members, but not gonadotropins or estradiol. Biol Reprod 70: 1828-1835, 2004.

28. Pedersen T and Peters H: Proposal for a classification of oocytes and follicles in the mouse ovary. J Reprod Fertil 17: 555-557, 1968

29. Weihua Z, Saji S, Makinen S, Cheng G, Jensen EV, Warner M and Gustafsson JA: Estrogen receptor (ER) beta, a modulator of ERalpha in the uterus. Proc Natl Acad Sci USA 97: 5936-5941, 2000
30. Shimakage M and Sasagawa T: Detection of Epstein-Barr virusdetermined nuclear antigen-2 mRNA by in situ hybridization. J Virol Methods 93: 23-32, 2001.

31. Bai T, Tanaka T, Yukawa K, Maeda M and Umesaki N: Reduced expression of death-associated protein kinase in human uterine and ovarian carcinoma cells. Oncol Rep 11: 661-665, 2004.

32. Bai T, Tanaka T, Yukawa $\mathrm{K}$ and Umesaki N: A novel mechanism for acquired cisplatin-resistance: suppressed translation of deathassociated protein kinase mRNA is insensitive to 5-aza-2'deoxycitidine and trichostatin in cisplatin-resistant cervical squamous cancer cells. Int J Oncol 28: 497-508, 2006.

33. Itoh N, Yonehara S, Ishii A, Yonehara M, Mizushima S, Sameshima M, Hase A, Seto Y and Nagata S: The polypeptide encoded by the cDNA for human cell surface antigen Fas can mediate apoptosis. Cell 66: 233-243, 1991.

34. Hengartner MO: The biochemistry of apoptosis. Nature 407: 770-776, 2000

35. Suda T, Takahashi T, Golstein P and Nagata S: Molecular cloning and expression of the Fas ligand, a novel member of the tumor necrosis factor family. Cell 75: 1169-1178, 1993.

36. Suda T and Nagata S: Purification and characterization of the Fas-ligand that induces apoptosis. J Exp Med 179: 873-879, 1994. 